\title{
Labyrinthe
}

$10 \mid 2001$

Numéro 10

\section{Le web et la culture : les start up and down}

Entretien réalisé par Mathieu Rigo et Sophie Laibe

\section{Frédéric Grolleau}

\section{(2) OpenEdition}

Journals

Édition électronique

URL : http://journals.openedition.org/labyrinthe/1192

DOI : $10.4000 /$ labyrinthe. 1192

ISSN : 1950-6031

Éditeur

Hermann

\section{Édition imprimée}

Date de publication : 1 octobre 2001

Pagination : 9-26

\section{Référence électronique}

Frédéric Grolleau, «Le web et la culture : les start up and down », Labyrinthe [En ligne], 10 | 2001, mis en ligne le 07 juin 2006, consulté le 30 avril 2019. URL : http://journals.openedition.org/labyrinthe/1192 ; DOI : 10.4000/labyrinthe.1192

Ce document a été généré automatiquement le 30 avril 2019.

Propriété intellectuelle 


\section{Le web et la culture : les start up and down}

Entretien réalisé par Mathieu Rigo et Sophie Laibe

Frédéric Grolleau

Frédéric Grolleau

Locaux spacieux, loft en open space, ordinateurs rutilants, ambiance « jeune »... notre entretien à Manuscrit.com avec Frédéric Grolleau, professeur de philosophie en détachement et figure emblématique de la critique littéraire sur le Web, a été l'occasion de faire un point - décontracté sur les espoirs et les désillusions de la « révolution » Internet, un média qui n’a pas fini de bousculer le domaine de la culture...

1 Labyrinthe - Comment vous définiriez-vous, au vu de vos différentes fonctions, vous qui avez participé au lancement de beaucoup de start-up, notamment, je lance le terme, de "start-up littéraires ». Quelques propositions: "pigiste multicarte ", " animateur de télé alternative ", peutêtre "confidentielle», et/ou en tout cas journaliste dans un média alternatif; peut-on considérer qu'il s'agit d'un média alternatif?

2 Frédéric Grolleau - Le mot alternatif oui, journaliste, c'est là où ça se complique ; il faudrait définir le terme. C'est le propre du philosophe de définir les concepts... au sens premier du terme, le journaliste est quelqu'un qui informe avec des chroniques journalières, ou des revues-papier, -plastique... c'est pour cela que je ne suis pas journaliste ; enfin je l'étais jusqu'à il y a assez peu de temps (septembre 2001), parce que je n'écrivais quasiment que sur Internet, si on laisse de côté le travail que je fais dans les revues.

3 Mes activités sur Internet sont principalement : des critiques de livres sur Paru.com, site qui date de 1998 (amorcé en 1997) ; ce n'était pas du journalisme, pas du billet d'humeur, c'étaient des présentations d'ouvrage; je ne suis pas journaliste, je n'ai pas fait d'école 
pour cela ; mais on me donne cette étiquette car j'ai travaillé pour des revues-papier, des articles de livres, voire des articles qui peuvent s'apparenter à du travail de journaliste...

Labyrinthe - Journaliste plutôt dans le sens "d'écrivant dans les médias", dans un sens vague... Vous écrivez sur votre créneau, puisque de formation littéraire, philosophe, vous avez un titre à parler de cela - et vous avez un média, ce qui n'était pas forcément dans vos plans au début? (Enfin, si l'on a un plan quand on exerce l'activité d'enseignant)...

$5 \quad$ Frédéric Grolleau - Ce serait prétentieux de dire que j'avais prévu il y a quelques années de travailler sur de multiples supports ; c'est le propre d'Internet : un support en appelle généralement un autre, par ramification, rhizome, capillarité; je suis passé assez rapidement sur Paru.com alors que j'étais encore professeur de philosophie en classes prépas, puis à la télévision sur Internet, c'était l'aventure CanalWeb, ce qui est d'autant plus touchant qu'ils ont déposé le bilan hier après-midi - quelque chose qui n'est pas sans m'interpeller et un peu m'inquiéter, sur ce que sont justement les nouveaux médias. C'est le signe en tout cas que ces nouveaux circuits de culture sont soit mal gérés, c'est en partie le cas de CanalWeb, soit peut-être encore refoulés, pour parler en termes psychanalytiques, par une partie de la population, ne serait-ce que parce que cette partielà n'y a pas accès ou ne veut pas y accéder, ou y accède avec des contraintes techniques ou technologiques qui le rebutent.

Labyrinthe - Pour CanalWeb, avant la fermeture, il était question de faire payer les prestations; y a-t-il un problème de rentabilité?

Frédéric Grolleau - Oui ; c'est un modèle économique qui n'est pas sans interroger, ou mettre l'accent sur un certain mode de fonctionnement.

8 Labyrinthe - Pour en revenir à vous, il semble qu'il y ait une sorte de contradiction implicite dans vos activités, entre le fait d'être chroniqueur littéraire (plutôt que journaliste ?)...

Frédéric Grolleau - Je ne suis pas chroniqueur littéraire non plus !

Labyrinthe - Vous refusez de vous définir en quelque sorte?

11 Frédéric Grolleau - Non au contraire, je me définis très précisément - Paul Valéry disait que l'indéfinissable faisait partie de la définition de la poésie - je suis critique littéraire. C'est un grand sujet de débat justement dans ces murs (Manuscrit.com), où je suis responsable éditorial, parce qu'on est plusieurs responsables éditoriaux (cinq). Il y a un secteur sciences humaines, histoire, très lourd à gérer, parce qu'on travaille beaucoup avec des universités, etc., on récupère des sujets de mémoires de maitrises, de thèses ; et à côté, il y a le pôle fiction, non-fiction, et je travaille avec Philippe Di Folco qui est responsable de la culture sur Nova, le site, la radio, la petite revue, qui travaille sur de multiples supports, sur Paris Première avec Frédéric Beigbeder, et lui se définit comme un chroniqueur littéraire, c'est-à-dire quelqu'un qui met en avant une subjectivité, dans des articles toniques, mais assez courts, sur des ouvrages. Moi au contraire, je revendique le titre de critique littéraire, c'est-à-dire quelque chose qui n'est pas que la simple présentation d'un texte mais un engagement analytique ou conceptuel un peu plus élaboré, fût-ce sur des bandes dessinées ou des DVD, que je couvre aussi. Peut-être que mon écriture n'est pas en ce sens une écriture Internet c'est-à-dire une écriture brève, pour des articles tenant sur un écran, de l'ordre de 1500 signes. Les chroniques sont peutêtre «trop longues », mais c'est la définition de l'audace, savoir jusqu'où on peut aller trop loin ; j'excède volontairement, en partie, les contraintes d'Internet. 
Labyrinthe - "Vous dépassez les bornes » de la définition de la culture, aussi, apparemment, dans son acception élitiste, puisqu'il n'y a pas de support qui ne vous intéresse pas ; par exemple, "l'humeur du marcassin "1 traite de la BD, vous êtes critique de BD... Donc c'est une définition assez large, actuelle d'ailleurs, de la culture. Vous voyez-vous dans la mouvance ou la suite de Nova, dans la filiation des radios libres?

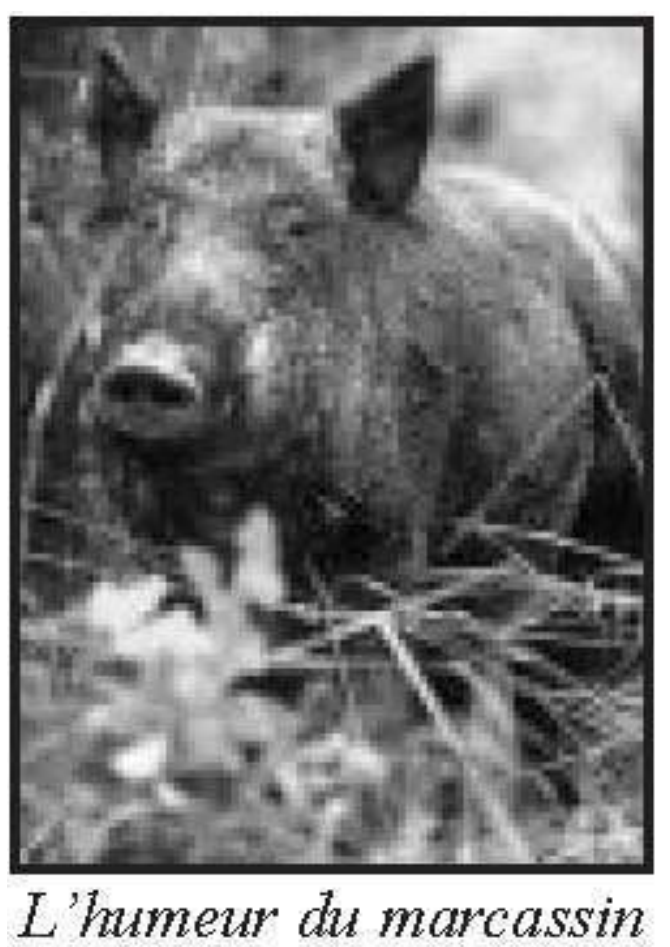

Frédéric Grolleau - Non ; peut-être vu de l'extérieur, cela fait penser à une filiation, mais en fait, plus simplement, je n'ai pas choisi de devenir cela, c'est l'ensemble de ces choses qui font que je suis passé de l'une à l'autre, mais je n'y suis pas passé de manière rayonnante en étant appelé, avec des salaires monstrueux; c'est un parcours modeste, banal, voire médiocre, au sens latin du terme : c'est un moyen terme, ce n'est pas quelque chose de si tranché que cela en fait.

Moi ce que j'essaie de faire, et c'est là que ça se complique, c'est utiliser la culture qui est la mienne, qui a ses lacunes comme celle de tout un chacun, pour éclairer d'un œil qui est le mien, des ouvrages qui m'intéressent. Rarement d'ailleurs les ouvrages qui me rebutent car quand un livre ne me plaît pas - sauf si je suis vraiment déçu, car la déception est une sorte d'amour inversé, c'est-à-dire que j'ai cru en ce livre, j'ai commencé à le lire, je me suis investi, j'ai été déçu, auquel cas je peux être assez sévère - je préfère ne pas en parler. Il y a suffisamment de bons livres pour laisser les mauvais de côté, il est inutile de faire de la polémique stérile. Il est très rare que j'écrive de manière négative ce qui est plus de la chronique littéraire; je ne veux pas faire comme certains magazines ou journaux, du type Les Inrockuptibles qui se sont fait une griffe, presque un label, dans la destruction; c'est toujours plus facile de démonter que de construire quelque chose, alors, j'essaie plutôt de construire.

Labyrinthe - Donc cela renvoie, en tout cas, par rapport au champ médiatique, comme vous l'évoquiez, à une perception de la culture qui est assez différente. N'y a-t-il pas une sorte de décalage entre l'activité hautement intellectuelle de décortiquer un roman ou un ouvrage, par rapport à l'analyse de quelque chose qu'on pourrait considérer comme de la « sous-culture »? 
16 Frédéric Grolleau - Il est évident qu'une BD n'est pas La Critique de la raison pure, qui ellemême n'est pas un essai sur le racisme... Ce sont des supports différents, on ne lit pas une bande dessinée de la même manière qu'on lit un roman ou un ouvrage de philosophie ; il est entendu qu'en voie de conséquence l'article n'est pas le même, et on ne s'adresse peut-être pas au même public.

17 Labyrinthe - Mais vous avez justement une espèce de relation différente avec le public, du fait du média employé ? Le « retour » sur le critique de BD n'est pas celui des critiques sur papier.

Frédéric Grolleau - Oui ; c'est peut-être utopique mais l'idéal serait pour moi, non pas de parasiter (car c'est un mot négatif), mais de créer des passerelles entre ces gens ; bien sûr, cela reste de la critique de $\mathrm{BD}$, je ne vais pas appliquer la théorie platonicienne à une BD de «Blake et Mortimer »; on peut le faire, ce serait d'ailleurs très intéressant, mais ce n'est pas l'objectif. L'objectif est plutôt de proposer un regard sur... Mais c'est un regard empreint de subjectivité, qui peut être déformé, et pas informé par une formation culturelle qui vaut ce qu'elle vaut. Vous parlez de «l'humeur du marcassin ", j'en suis flatté car c'est un site relativement peu connu du public, parce que j'ai tout fait pour ; je ne lui ai pas donné le nom d'un site de critique littéraire, pourtant à ma connaissance, c'est un des rares sites de critique littéraire existant; c'est ce qui m'a intéressé dans Internet, c'est la possibilité d'un renversement : en fait, je n'ai pas écrit des articles pour les offrir à des gens et dire : regardez-moi. Il m'est arrivé, par le passé d'écrire des articles sur de multiples supports parce que cela m'intéressait et un jour, je les ai regroupés en une « BDP », une base de données personnelle. Ce sont mes tiroirs à moi ; il y a une phobie chez les journalistes et chroniqueurs du Web, parce que l'on est dans le virtuel, pas dans le palpable, c'est de la pixellisation, alors on imprime « comme des fous " nos textes pour les archiver...

19 Je me suis rendu compte que j'avais un outil extraordinaire dans Internet; il suffisait de recollecter l'ensemble de ces textes et de les archiver dans un endroit si possible arbitraire. "L'humeur du marcassin » est d'ailleurs un site professionnel au plus haut point, puisqu'il est ouvert aux attachés de presse, aux éditeurs, etc.

20 Pour donner un exemple concret, en termes d'interactivité, dans la presse traditionnelle et dans le monde des revues aussi, les délais sont assez lourds, puisqu'il y a le bouclage, la mise sous presse, la distribution qui n'est pas très facile (notamment pour les revues) ; en fait, l'ensemble de ces supports prend du temps à mettre en place et à être offert au public. L'avantage c'est que moi, si par exemple aujourd'hui je vois une information ou même dans une librairie un livre qui me plaît, le fonctionnement est simple : j'appelle l'attaché de presse à qui je fais confiance pour le traitement des ouvrages, et je lui demande de me l'envoyer. On m'envoie l'ouvrage, et si je suis «énervé », je le lis dans la nuit - quel que soit son format, - j'écris un article immédiatement et le lendemain matin, cela prend deux minutes de mettre cet article en ligne sur mon site, ou il est envoyé à Paru.com (par le passé), Amazon aujourd'hui, Lire... C'est très rapide, très réactif ; c'est de la réactivité, pas au sens de Nietzsche, mais une valeur positive en l'occurrence.

21 Labyrinthe - À propos du réactif, il y a justement la question du retour du public. Paru.com fonctionne sur un principe que vous appelez la plate-forme; vous avez parlé de passerelle tout à l'heure; vous mettez en place une sorte de trilogie éditeur-lecteur-auteur que vous chapeautez. Dans cette configuration originale, le rôle de l'auteur est revalorisé car selon le directeur de Paru.com, " on n'est pas là pour servir la soupe, mais pour affronter un texte ». Est-ce qu'il n'y a pas là une dimension d'utopie par rapport à l'édition traditionnelle? 
Frédéric Grolleau - Il faut bien séparer les choses : officiellement, je suis responsable éditorial ici à Manuscrit.com, qui est une maison d'édition multi-supports; tournée vers différentes activités, assez révolutionnaire dans le marché français. Paru.com c'est un site de critique littéraire où je ne travaille quasiment plus puisque l'utopie a dégénéré : la qualité de ce site reste ce qu'elle est, mais les problèmes de financement ont fait qu'au bout d'un certain temps, c'est-à-dire en gros au bout de trois cents articles non payés et 50 heures par semaine pendant deux ans, j'ai estimé que je pouvais maintenant décemment, ou indécemment, espérer gagner ma vie en vendant mes articles, c'est-à-dire en devenant multi-pigiste. Ce site m'a lancé en tant que critique littéraire, et m'a lancé tout court sur Internet, mais dans des conditions qui seraient maintenant complètement hallucinantes si on les décrivait. C'était du bonheur pur à $150 \%$, avec des universitaires ou des professeurs qui ont simplement envie d'écrire, et de faire partager une passion..

Labyrinthe - C'était un peu la grande époque?

Frédéric Grolleau - Oui, depuis il y a eu le crash ; il y a eu soit le financement de ces sites, qui sont devenus autre chose; soit le départ forcé, ou finalement consenti des gens qui animaient ces entreprises, ou la disparition pure et simple. C'est aussi le danger de tout ce qui touche à Internet; il ne fait pas bon créer une start-up sur Internet à l'heure actuelle, surtout dans le domaine de la culture, qui représente un pourcentage infime.

Labyrinthe - Alors que pourtant, Paru.com a eu une plus longue durée de vie que Bol.fr : c'est le Web commercial qui a été sabré par la crise, alors que la curiosité du lecteur éventuel sur Internet reste encore satisfaite.

Frédéric Grolleau - Oui, en ce sens, le bénévolat sur Internet est quelque chose d'assez fabuleux; mais on peut comprendre qu'économiquement cela ait aussi une validité, puisque quand vous ne payez pas de charges, pas de frais de locaux, et pas de personnel, vous pouvez espérer durer plus longtemps qu'une entreprise comme CanalWeb, qui dépensait quand même 150 millions de francs par an.

Bien que j'aie de l'estime pour Patrick Lienhardt qui dirige le site de Paru.com, on ne peut pas rester éternellement dans un modèle qui mime le bénévolat heureux, quand on a quand même vendu un site pour 3 millions de francs, sans qu'il y ait de retour pour les personnes qui l'ont créé. L'enthousiasme oui, mais l'exploitation débridée, non. Il faut savoir s'arrêter. Mais une fois de plus j'aurais vraiment adoré pouvoir conserver les fonctions qu'on m'avait promises au départ, qui m'avaient fait travailler comme un fou et construire en bonne partie à moi tout seul, car un quart de la base de données vient de moi (romans, essais, BD, philosophie...), j'aurais aimé rester responsable pour la littérature, comme je l'étais pendant deux ans.

Labyrinthe - Donc c'est pour vous personnellement, et aussi peut-être pour l'économie d'Internet, l'économie de la culture sur Internet, l'heure de ce que vous appelleriez maison d'édition "révolutionnaire ", c'est-à-dire de nouvelles solutions d'édition...

Frédéric Grolleau - Oui, il y a eu trois axes, si on veut reprendre cela historiquement; il y a eu Paru.com, qui continue, et il y a d'ailleurs une nouvelle version remarquable du site, encore mieux achalandée, très diverse, avec des papiers très bien construits, denses, que je conseille ; puis il y a eu à cheval l'aventure CanalWeb, la fusion des deux ayant donné Paru.TV, qui était une série d'émissions littéraires hebdomadaires que j'ai animées pendant deux ans ; au total presque une centaine d'émissions en direct avec le gratin de la littérature française voire européenne...

Labyrinthe - qui était d'ailleurs demandeur, très intéressé... 
31 Frédéric Grolleau - Début 99, quatre ou cinq émissions devaient être faites, que personne ne voulait animer bénévolement, pendant un an - maintenant cela fait sourire les gens, qui se précipiteraient sur l'occasion - mais à l'époque personne ne voulait le faire; je l'ai fait, mais sans avoir au préalable initié une démarche pour devenir journaliste TV; au final j'étais ravi de cette activité. J'ai donc fait ces émissions pour CanalWeb qui étaient le fer de lance et la griffe de Paru pendant très longtemps, puisqu'il $\mathrm{y}$ avait un espace où on pouvait toujours consulter ces entretiens, conservés également dans «l'humeur du marcassin » en ligne pour la plupart, comme une sorte de musée personnel.

Puis j'ai senti le vent tourner, en avril 2001 ; la direction de CanalWeb voulait de plus en plus commercialiser, gagner de l'argent, et cela voulait dire pour eux, à terme, s'intéresser uniquement à des modes payants et très "people » si l'on peut dire, de programmes (plus très) culturels. Donc cela ne m'intéressait pas de traiter uniquement DU livre à la mode, du énième ouvrage pornographique dont parlait toute la presse ; j'ai préféré partir.

Le hasard, une fois de plus, ou la ramification, m'a permis de rencontrer les gens de Manuscrit.com qui m'ont proposé de participer à cette entreprise qui était naissante aussi, et voilà... Mais ce ne sont jamais des choses prévues, et préméditées... Labyrinthe - vous surfez, en quelque sorte?

Frédéric Grolleau - Je ne suis pas un excellent surfer, parce que j'ai peur de tomber... mais c'est aussi comme cela qu'on remonte sur la planche...

Labyrinthe - Mais vous commencez à vivre de cette activité?

Frédéric Grolleau - Officiellement, je suis en disponibilité pour convenance personnelle, chèrement acquise auprès du rectorat de l'académie de Créteil, depuis un an, pour une période de six ans. Je me suis battu comme un chien pour obtenir ce droit, quand j'ai fait cette demande, d'abord neuf mois à l'avance, et ma hiérarchie l'a refusée à trois reprises, malgré dix ans d'enseignement, y compris dans les zones sensibles, difficiles ou très violentes, j'ai dû devenir professeur de boxe française pour survivre, j'ai dû me battre, m'adresser directement au chef de cabinet du nouveau ministre de l'Éducation nationale d'alors, Jack Lang, en lui expliquant que je pouvais très bien être mis en détachement pour le ministère de la Culture; de fait c'est ce que je faisais. J'ai obtenu l'appui de la République à l'arrachée, à tel point qu'à trois jours près, j'étais banni, rayé de l'Éducation nationale, comme un malpropre. Ce n'était pas une partie de plaisir. Mon poste est donc « gelé » au lycée de Savigny-le-Temple, mais j'espère y retourner le plus tard possible... voire jamais.

\section{Labyrinthe - Gardez-vous un mauvais souvenir de vos années d'enseignement?}

Frédéric Grolleau - Non, j'ai toujours eu plusieurs activités, j'ai été un «polyprofesseur » (avec un Y à poly) ; j'ai à la fois de très mauvais et d'excellents souvenirs, j'ai fait cinq académies, je suis passé du Nord au Sud, de Maubeuge à Aix-en-Provence, et des plus grands lycées de France (comme le Prytanée de la Flèche) aux plus mauvais...

Labyrinthe - Vous êtes content d'avoir tourné la page.

Frédéric Grolleau - Je n'ai pas tourné la page définitivement, mais je suis content de faire autre chose, pour l'instant.

Labyrinthe - Vous restez toujours dans une optique de transmission de la culture... 

que les professeurs de philosophie, malgré leur bonne volonté, ne font rien si le public n'est pas réceptif un minimum; et de plus en plus j'ai rencontré un public de moins en moins réceptif... malgré les cours de philosophie axés sur le cinéma, la $\mathrm{BD}$, la culture, des choses basiques, « sous-culturelles » comme vous le disiez; mais je suis un peu désespéré par rapport à cela. C'est vrai que je m'étais un peu émoussé, j'y croyais un peu moins, cela se voyait ; j'ai préféré non pas tourner la page mais lire un autre livre, passer à une autre page.

Labyrinthe - Vous avez évolué vers Manuscrit.com sur le Salon du livre; ils vous ont rencontré...

Frédéric Grolleau - Exactement. Le principe était assez extraordinaire, parce qu'une maison d'édition, en France, qui conjugue à la fois un volet Internet très fort et en même temps un fonctionnement somme toute traditionnel, tout en passant du livre format PDF, au fichier téléchargeable, par l'impression à la demande jusqu'aux livres traditionnels papier, puisque officiellement Manuscrit.com se dédouble d'une structure qui s'appelle Nicolas Philippe, qui devrait faire paraître ses premiers titres en février sous ce label (certains titres sont déjà parus en coédition)...

Labyrinthe - Nicolas Philippe c'est le «Serpent à Plumes»?

Frédéric Grolleau - C'est lui qui a créé Manuscrit.com, il détient aussi le "Serpent à Plumes ", «Florent Massot Présente » et Somogy (livres d'art), un groupe qu'on appelle les éditions du Forum... tout en étant vraiment indépendants, au niveau ligne éditoriale, $\mathrm{du}$ financement, et du concept justement... donc ce qui m'a intéressé évidemment làdedans, c'est que Manuscrit.com renverse la chaîne du livre : dans la plupart des cas, pour faire bref, les libraires et les revues littéraires deviennent des réceptacles d'ouvrages qui sont formatés, qu'on leur distille, ici, c'est l'inverse. On reçoit un manuscrit, sur une base de données, sur Internet uniquement, en fichier joint comme dans un e-mail ; on envoie ce manuscrit, après un premier travail de filtre accompli par le conseil éditorial et les internautes en tant que tel, on envoie ce manuscrit à tous nos partenaires libraires, revues, etc. - dont Labyrinthe fait partie ! - et dans plus de 21 genres, l'érotisme, la science-fiction, les sciences humaines pures et dures... et ces manuscrits nous reviennent enrichis d'une lecture, qui n'est pas uniquement la vente de soupe, une fois de plus, mais les qualités, etc., les défauts...

Évidemment nous, grâce à ces informations-là, on fait plusieurs choses avec ce texte : dans le meilleur des cas, ça devient un livre-papier que nous éditons; dans un cas intermédiaire, qui n'est pas infamant, c'est une coédition avec un éditeur dont la ligne éditoriale se rapproche peut-être plus du thème, et dans les cas qui restent, l'ouvrage, ne nous permettant pas d'espérer des ventes de nombreux exemplaires, est retravaillé, reformaté, et édité tout simplement, accessible en impression à la demande, avec des tarifs dégressifs selon la quantité...

Labyrinthe - Un type d'édition qui fait l'objet d'une véritable demande du public...

Frédéric Grolleau - Le truc assez faramineux, je peux le dire en toute objectivité étant auteur moi-même...

Labyrinthe - C'est une espèce de narcissisme universel qui trouve à s'exercer, une variante de la volonté de pouvoir que recélait pour Kundera la vogue des journaux intimes... 
52 Frédéric Grolleau - Ce qu'il faut bien dire en tout cas, c'est que n'importe quelle personne qui écrit ne serait-ce qu'une petite nouvelle (puisqu'on a un seuil minimal qui doit commencer je crois à trente-cinq mille signes, le format d'un article de revue), peut envoyer son texte à Manuscrit.com et la procédure est très simple, on envoie un fichier Word, par exemple, vous vous inscrivez sur Manuscrit.com, ce qui vous donne un identifiant, tout cela est gratuit... le texte est immédiatement enregistré chez nous, s'il ne contrevient pas aux lois minimales de déontologie et d'éthique, il reçoit automatiquement un numéro d'ISBN, et pendant dix-huit mois il devient propriété de Manuscrit.com.

Labyrinthe - C'est une couveuse...

Frédéric Grolleau - Voilà. C'est aussi un délai très court, parce que la plupart des maisons d'édition, quand elles mettent le grappin sur un texte, le gardent ad vitam aeternam et n'envisagent pas de le sortir avant deux ou trois ans... donc dix-huit mois, il faut qu'on fasse nos preuves, qu'on vende, qu'on fasse la promotion dans le bon sens de ces termes, de ce texte auprès de nos libraires, nos critiques, etc. Moi j'aurais rêvé de ça il y a dix ans : j'étais un écrivaillon, ce que je suis encore d'ailleurs, j'écris des articles assez fréquemment qui sont lus par un lectorat très réduit, la revue Res Publica, dont je suis rédacteur en chef adjoint, c'est une revue qui avant que les PUF nous donnent un petit coup de main, était éditée un peu comme Labyrinthe à cinq cents exemplaires... on en était heureux... être lu par cinq cents personnes...

Labyrinthe - Oui, cinq cents exemplaires, ce n'est pas forcément cinq cents personnes...

Frédéric Grolleau - Oui, on peut rêver... donc il y a des mutations qui font que, quand vous déposez un texte, vous devenez un auteur. Et ce texte devient un livre, un ouvrage, objectivable, matériellement, une couverture, votre nom, si votre narcissisme vous a conduit jusqu'à utiliser votre propre nom, avec des droits d'auteurs, sur le fichier, l'impression à la demande...

Labyrinthe - Alors justement, il y a eu dans le domaine de l'Internet de grosses polémiques sur cette question des droits ${ }^{2}$.

Frédéric Grolleau - Ici c'est très simple, il suffit d'aller en ligne pour s'en rendre compte, dès qu'un texte est déposé, il y a un numéro d'ISBN qui fait que si quelqu'un utilise les données de ce texte il pirate un ouvrage déposé, auprès de la Société des Gens de lettres, reconnu comme propriété intellectuelle de l'auteur.

Maintenant, ça ne veut pas dire que cet ouvrage est un chef-d'œuvre, qu'il deviendra le Houellebecq de demain... mais c'est la première fois dans l'histoire qu'en des délais si courts, un ouvrage devient une œuvre à part entière. Même si cette œuvre devra faire son chemin, devenir quelque chose d'autre peut-être, on fait un travail aussi d'élaboration, de modification, pour justement faire éditer ce texte idéalement en version papier, puisque nous visons cette forme malgré tout... c'est à la fois une librairie, un club, puisque nous sommes agents littéraires au sens large, nous mettons en relation des auteurs avec d'autres, dans tous les genres ; c'est évidemment, une maison d'édition, mais avec un volet interactif très puissant, puisqu'on ne travaille qu'à base de courriers électroniques...

Labyrinthe - Vous avez en quelque sorte opéré un renversement d'ordre économique: de l'économie de l'offre à l'économie de la demande.

Frédéric Grolleau - Oui mais ce n'est qu'une partie de la révolution... parce qu'il y a une partie des ouvrages que nous coéditons, sans d'ailleurs malheureusement gagner de 
l'argent sur ceux-là, mais sans en perdre non plus, en espérant que la possibilité qui va être donnée sur tel recueil de poésie ou tel essai historique permettra, peut-être, de trouver un texte plus "intéressant», qui vendra plus d'exemplaires et, du coup, rétribuera la structure et le fonctionnement.

Frédéric Grolleau - Oui, vous avez vu le logo ? C'est une icône sumérienne qui désigne l'écriture...

Labyrinthe - À votre avis - si tant est que dans le domaine d'Internet on puisse faire des pronostics: je ne sais pas si il y a deux ans vous auriez fait le pronostic de ce qui a eu lieu entretemps, la fin de la bulle spéculative, etc. - par rapport à ce que l'on disait à ce moment-là, est-ce que ces médias paraissent encore porteurs de développement, autonome d'ailleurs par rapport aux autres médias, peuvent faire naitre une économie de la création, par exemple, quelque chose de cet ordre, ou est-ce qu'au contraire, l'utopie dure encore un peu mais va... tomber? Question qui en appelle une autre : comment arrivez-vous à vous intégrer dans les circuits traditionnels? Est-ce qu'ils vous respectent en tant que " nouveaux » médias, canaux...

65 Frédéric Grolleau - Alors [se tournant vers l'un puis l'autre intervieweur] je réponds peutêtre à votre question, et à la vôtre, dans l'ordre... nous sommes perçus de deux façons, assez évidentes, je crois, comme toute structure new age révolutionnaire qui se respecte... comme par exemple Amazon ou Bol qui se sont installés, on diabolise un peu le nouveau venu, qui forcément marche sur les plates-bandes des monopoles existants... vieille histoire qui est déjà dans la Bible, c'est la logique du tiers exclu... on est mal perçus par certains éditeurs, qui, en fait, ont très bien compris l'intelligence du projet, j'emploie le mot à dessein, selon l'étymologie, qui renvoie à cette position de liens qui se relient entre eux et mettent en fait en apposition des secteurs qui étaient séparés... ils n'ont pas eu l'idée, ou les moyens : je devrais écrire un jour un livre ou une petite nouvelle sur la manière dont Internet a été vécu par les éditeurs traditionnels, et notamment par les attachés de presse... je me rappelle des journées entières où j'expliquais comment utiliser un clavier, le courrier... évolution qui est assez remarquable !

Cela étant, on est aussi bien appréciés, parce qu'on offre des services qui sont intéressants, parce qu'on offre de choses qui sont inédites, et une fois de plus, pas payantes, qui permettent à nos partenaires d'être mis en avant.

Labyrinthe - En termes de stratégie, c'est payant pour vous, aussi...

8 Frédéric Grolleau - Pas dans l'immédiat, mais à terme... aujourd'hui, il y a de petits éditeurs qui existeraient moins, moins bien, sans nous. Les gros commencent à nous faire de l'œil, Lattès, avec qui nous sommes depuis longtemps en partenariat, nous sommes en tractations avec Flammarion ; Grasset nous a refusé plusieurs fois des manuscrits, de bonne qualité objective, parce qu'il était trop tôt... on est dans cet entre-deux, entre la vie et la mort [rire]...

Pour revenir à votre question, je ne suis pas Nostradamus, mais il est clair qu'aujourd'hui Internet ne représente plus ce que ça représentait, la folie dot.com... il y a d'ailleurs un excellent ouvrage sur ce point, Totalement inhumaine de Truong... chroniqué sur "l'humeur du marcassin $»^{3}$ un ouvrage que je conseille vraiment à tout le monde... c'est un grand auteur français, malheureusement trop méconnu... enfin, Internet n'a plus la cote qu'il avait... cela dit, on voit bien en termes de broadbanding, de webcast etc., tout ce qui anime les plates-formes, tout cela a un bel avenir devant soi, mais si justement elles 
s'associent avec des portails existants. Ça a été l'erreur, je pense, de CanalWeb, de vouloir être freelance...

Labyrinthe - Dès qu'on parle Internet, on parle en anglais, vous avez remarqué?

71 Frédéric Grolleau - Les noms vont se franciser, mais c'est une culture qui vient des ÉtatsUnis quand même... je pense que ce qui s'est passé tient à ce qu'une des clauses d'Internet n'a pas été respectée, celle de l'association. Pas forcément intéressée, mais le partage en fait... CanalWeb aurait pu durer davantage en association avec Voilà ou Wanadoo... il faut savoir qu'à l'époque ça ne s'est pas fait, pour des raisons économiques, parce que quand on parle d'Internet on parle d'économie, à l'époque France Télécom a freiné des quatre fers, parce qu'on ne pouvait pas laisser se développer un nouveau média qui lui aurait échappé. Ce n'est pas un hasard si à l'heure où Voilà est devenu le portail que l'on sait, où Wanadoo rayonne, et où ils devraient incessamment devenir payants, à mon avis, ce n'est pas un hasard si le développement de ces deux sites coöncide avec la déréliction de CanalWeb. Il devrait se passer peu de temps avant qu'on voie des images TV diffusées sur ces sites, mais avec des plates-formes promos étatiques, etc.

Labyrinthe - Avec une espèce de marchandisation, d'homogénéisation des contenus offerts... une télé classique...

Frédéric Grolleau - Ce qui est presque fatal, mais ça a été aussi l'erreur de CanalWeb, et d'autres sites, parce que les plates-formes utilisées sur le Web ne fonctionnent pas terriblement, ça a été de croire qu'on pourrait lancer une nouvelle culture indépendamment des contraintes financières... et puis aussi, il est évident que cette offre est venue trop tôt, ce n'est pas comme la philosophie, qui vient toujours trop tard, il est clair que l'internaute moyen n'est pas équipé de matériel suffisamment sophistiqué, et si jamais il l'était, l'accès était encore trop compliqué. C'est un tout, à ne pas méconnaître, qui a produit une forme d'élitisme...

Labyrinthe - Élitisme dans lequel vous vous retrouviez, d'une certaine façon, puisque servir la culture, et non la soupe... enfin, à condition que les deux cas de sélection se recoupent...

Frédéric Grolleau - C'est peut-être là où il y aurait une contradiction, puisque le support Internet prétend universaliser des connaissances, alors que le médium qui met en avance ces connaissances présuppose une forme d'élitisme technologique... cela dit, il y a un effort qui a été fait depuis environ deux ans, par l'Éducation nationale, qui met à disposition des établissements des connexions libres pour les élèves et les étudiants... enfin, ça vaut ce que ça vaut, mais ça a le mérite d'exister... Il existe des sociétés qui proposent des ordinateurs pratiquement gratuitement, pendant deux ou trois ans, à condition qu'on paye un abonnement à Internet de $200 \mathrm{~F}$ par mois... on n'est plus obligé d'investir dans le matériel $30000 \mathrm{~F}$ pour surfer à haut débit... le marché se régule aussi par rapport à cela, mais il est évident que Manuscrit.com illustre, disons, cette frontière entre les nouvelles technologies et des lecteurs traditionnels... cela dit, la plupart des gens ont un courrier électronique, et à bien regarder, n'en demandent pas plus dans la plupart des cas... déjà pour envoyer un manuscrit en pièce jointe, ce n'est pas non plus l'Everest technologiquement parlant. Ça l'était il y a deux ans.

Ce ne sont pas que des jeunes, mais il y a des études qui montrent que ce sont pour beaucoup des retraités pour qui Internet est devenu un passe-temps quotidien, et il existe autant de tranches d'âge différentes qui s'intéressent à Internet... il y a des gens qui utilisent leur RTT pour pianoter, des écrivains plus ou moins confirmés, des journalistes au sens propre du terme, qui sont intéressés par ce nouveau vecteur et la possibilité enfin 
d'être lus, contrairement à ce qui se passe dans les maisons d'édition où, pour peu qu'il y ait un manuscrit qui tombe derrière, on l'oublie... ça n'arrive a priori pas chez nous : c'est l'avantage de la technique au service de la culture, le traitement est plus facile. La plupart des auteurs dont je m'occupe ici, je leur téléphone rarement, je les vois rarement, même s'ils habitent dans la rue d'à côté, mais je leur écris tous les jours.

Labyrinthe - Est-ce que le bain de culture où vous travaillez, et vos activités en général influencent votre écriture de romancier? Votre notice biographique sur Manuscrit.com annonce, sous auteurs favoris, "Kafka, Cervantes, Borges, Hergé » : est-ce que c'est un programme?

Frédéric Grolleau - Hergé est encore plus important que tout le reste... le mot romancier doit être nuancé, puisque jusqu'ici les ouvrages publiés sous mon nom sont principalement des essais philosophiques, au sens large, que j'ai écrits en tant que professeur en classe préparatoire, avant de devenir l'homme venu d'Internet dont vous parlez. Je suis en train de finir mon premier roman, c'est un scoop d'ailleurs, j'ai tout fait pour que ça ne se passe justement pas dans ce milieu-là. Il y avait ces deux écueils à éviter absolument, celui de parler de soi : il fallait que j'aille très loin et dans le temps et dans l'espace pour éviter cela, et celui d'évoquer le monde d'Internet, alors que j'ai eu des commandes pour ça. J'avais déjà un titre : Le Web est dans le canal. Mais ça, je refuse de le faire, même si j'aurais peut-être dû accepter, c'eût été tentant d'écrire un 99 francs sur le milieu d'Internet! Mais je n'envisage pas néanmoins de ne jamais le faire.

Labyrinthe - Est-ce qu'en tant que critique, vous ne vous sentez pas justement hyper-critique vis-à-vis de votre production?

Frédéric Grolleau - Une chose est sûre, c'est que quand on écrit, on s'écrit souvent. Il est difficile de se départir de processus inconscients, Dantec écrit de très belles pages làdessus, évoquant l'écriture comme travail de sape pour trouver un lieu et dépasser toutes ces strates. D'un point de vue conscient, je n'imagine pas être déformé par ça... peut-être qu'un jour, quelqu'un s'amusera, quand je serai mort, à relire toutes mes critiques, tous mes textes, et verra qu'il y a des points tristement récurrents... mais c'est le risque que je prends en donnant des articles qu'il suffit de copier/coller pour que des gens se voient payer $1500 \mathrm{~F}$ pour un texte, alors que cela m'en rapporte en moyenne 300. Il faut dire sans hypocrisie que si je ne voulais pas m'exposer ni à être critiqué, ni à être pillé, je n'aurais pas créé «l'humeur du marcassin», je donnerais des cours en lycée à SaintCloud.

81 Labyrinthe - Vous avez parlé de révolution au sujet d'Internet (qui se stabilise comme toute révolution) : cela est-il le cas aussi en termes d'écriture, du fait de l'absence des contraintes habituelles de temps, de taille...?

Frédéric Grolleau - Bon, c'est tout sauf du premier jet, mais ça s'apparente à quelque chose de cet ordre : je rêvais de faire ce que je fais aujourd'hui, plus jeune. Il y a quelques années que je n'ai pas acheté de livres... recevoir des ouvrages en échange simplement du point de vue que je pourrai donner là-dessus.

Labyrinthe - Le «métier de lire», bis ${ }^{4}$. On vous a comparé à un nouveau Pivot...

Frédéric Grolleau - Ça c'est une polémique, il y a eu un article très sympathique et élogieux, qui m'a projeté sur le devant de la scène en avril 2000 je crois, dans Le Monde, qui m'a appelé " le pivot du Web ", c'est évidemment très flatteur et en même temps... évidemment il y a le jeu de mots sur pivot... mais simplement, j'ai essayé de faire quelque chose avec le Web, dans le domaine des émissions de télé, qui était inédit. Aujourd'hui il y a peut-être dix personnes qui font cela, mais seulement à cette époque, c'était 
révolutionnaire : comment faire une nouvelle télévision littéraire ? Moi j’ai inventé cela, il y a déjà deux ans... c'était neuf en termes d'interactivité, il y avait un chat intégré, j'ai inventé un archivage, une télévision où, en même temps qu'on visionne l'émission, on a accès à toutes sortes d'articles de presse, à tous les liens sur l'auteur, enfin... sur le même écran, dans le même espace... et aujourd'hui le support principal, CanalWeb, va maintenant disparaître.

Alors soit ces programmes qui sont sur le site vont être repris, soit, et ce n'est pas exclu, ils vont disparaître eux aussi... pas dans l'espace de la Toile, mais c'est une question philosophique importante, de l'espace de la Toile. Je ne sais pas comment ces informations, captées par une sorte d'aspirateur géant, peuvent être assurées de perdurer.

\section{ANNEXES}

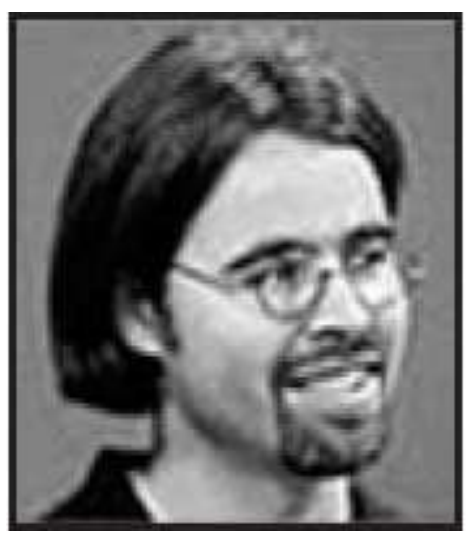

\section{NOTES}

1. http://www.webzinemaker.com/marcassin

2. Une indiscrétion a permis à un site commercial d'annoncer la prochaine parution d'ouvrages dont seuls les contrats étaient signés, le manuscrit à peine commencé. Un procès a suivi, intenté par Jean-François Revel.

3. http://www.webzinemaker.com/admi/m9/page.php3? num_web=1489\&rubr=2\&id=5053. Jean-Michel Truong, Totalement inhumaine, Les empêcheurs de penser en rond, 2001, 219 p.

4. Titre d'un ouvrage d'entretiens entre Bernard Pivot et Pierre Nora, récemment republié à l'occasion de la fin de l'émission « Bouillon de culture ». 